\title{
Review
}

\section{Hepatic Diseases in Canine and Feline: A Review}

\author{
Kassahun A. Negasee, DVM, MSc* \\ Department of Animal Health, Awi Zone Livestock Office, Amhara Region, Ethiopia
}

"Corresponding author

Kassahun A. Negasee, DVM, MSc

Specialist in Veterinary Medicine, Department of Animal Health,Awi Zone Livestock Office,Amhara Region, Ethiopia; E-mail: kassahunayana@gmail.com

\section{Article information}

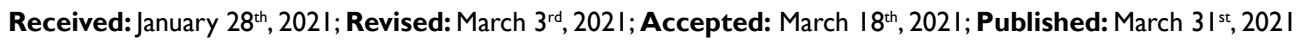

\section{Cite this article}

Negasee KA. Hepatic diseases in canine and feline:A review. Vet Med Open J. 202I; 6(I): 22-3I. doi: 10.17I40/VMOJ-6-I55

\begin{abstract}
Dogs and cats are belonging to canine and feline family respectively. The liver is the largest gland in the body and is located in the cranial abdomen between $3^{\text {rd }}$ and $4^{\text {th }}$ ribs in dogs and cats. This review is mainly focused on: to understand the anatomy and physiology of liver, the liver diseases pathophysiology, to diagnose the liver diseases, managemental and ameliorative methods of liver diseases. The dual blood supply to the liver is hepatic artery and portal vein. The function of liver includes the regulation of digestion and metabolism, the synthesis of hormones and proteins, immune response and filtering of toxins from the blood stream. Any problem that affects the liver is liver disease. Inflammation of liver is hepatitis. Hepatitis caused by infectious, non-infectious, auto-immune and reactive. It can be acute and chronic. The most encountered liver diseases in dogs and cats are hepticlipidosis, cholangiohepatitis, portosystemicshunt, cholelithiasis, choledocholithiasis, cholecystitis, pneumobilia and hepatic neoplsia. The clinical symptoms of liver diseases include jaundice, hepatic encephalopathy, gastro intestinal disorders and nonspecific signs include polyuria/polydipisia. The liver disease diagnosed based on history, liver function tests, medical imaging. The latest imaging procedures are endoscopic retrograde cholagio pancreatography (ERCP) and computed tomography (CT). For confirmatory diagnosis liver biopsy and histopathological interpretation is required. Therefore, based on diagnosis appropriate treatment should be selected: bile stasis is treated urodeoxycholic acid (URDA), fluid therapy include sugar and salt solution for replacement fluid loss, gastrointestinal protectors include ranitidine, cimitidine and lactulose, albumin to treat hypoalbuminimia, antioxidant for scavenging free radicals include vitamin E, Selenium, S-adenosyl-L-methionine (SAMe), diuretic fursimid for treatment of ascites and supplementation low protein diets. This laparoscopic technique for removal of gallstones and endoscopic retrograde cholagiopancreatography (ERCP) to treat gall stones on bile ducts and to widen the slipped ducts and liver transplantation for cirrhotic dogs and cats. Early accurate diagnosis and managing any predisposing factors that affects the health of dogs and cats are important for controlling the liver diseases.
\end{abstract}

\section{Keywords}

Canine; Feline; Liver disease; Hepatitis; Hepatocyte; Kuppfer cells.

\section{INTRODUCTION}

$\mathrm{D}$ ogs and cats are domestic animals belonging to different species; canine and feline families respectively. There are many differences between a dog and a cat including their physical features, nature and character. ${ }^{1}$ Both dogs and cats are our wonderful and lovable pets; canines and felines are friends more than anybody for human beings because of their keen observations, patience and easily adapt with people in the home. ${ }^{2}$

The dogs and cats like other animals for maintaining their life physiology have different organs and tissues among that is the digestive tract. Their function is digests food, absorption and eliminates wastes from the body. ${ }^{3}$ The liver is the main principal largest glandular digestive tract organ located under a rib cage on the right side in the abdominal cavity. ${ }^{4}$ It consists of hepatocytes, hepatic stellate cells or Ito cells and sinusoidal cells include kuppfer cells and endothelial cells. ${ }^{5}$

The major functions of the liver are carbohydrate, protein and fat metabolism, detoxification, secretion and storage. Thus, maintaining a healthy liver is a crucial factor for the overall health and well-being of life of animals. ${ }^{6}$ It is vital and complex organ of the body, it become susceptible to many adverse effects including drugs, chemicals, infectious agents, autoimmune disease, and reactive hepatitis and there is also idiopathic occurrence. ${ }^{7}$

The liver disease includes hepatocellular reversible and irreversible injury (necrosis), porto systemic shunt, neoplsia (primary hepatic and secondary) and hepatic fibrosis or cirrhosis. ${ }^{7}$ Pets 
with liver disease can present with arrangement of clinical conditions from severely ill to asymptomatic. Some vague signs can be depression, weight loss, jaundice, gastro-intestinal and neurological symptoms. ${ }^{8}$

The liver disease diagnosed by using history and physical examination, liver function tests and medical imaging techniques. However, for identification of specific hepatopathies definitive diagnosis of liver disease the histopathological examination is required to set recommended treatment. ${ }^{9,10}$ The hepatic diseases are managed by reducing predisposing factors, providing intravenous fluid to rehydrate, administering gastro-intestinal protectants to prevent ulcerations, avoiding the use of sedatives, supplementation of low protein diets and Zinc for regulation of protein and nitrogen metabolism, cholecystectomy and antibiotics are also used for management of hepatic diseases. ${ }^{10,11}$ Therefore, the objective of this review is to understand the anatomy and physiology of liver, the liver diseases pathophysiology, to diagnose the liver diseases, manage mental and ameliorative methods of liver diseases.

\section{THE LIVER ANATOMY AND PHYSIOLOGY}

\section{The Liver Anatomy}

The liver, that lies across the stomach and duodenum and derived from the embryonic gut. ${ }^{3}$ The liver is the largest gland in the body and is located in the cranial abdomen between $3^{\text {rd }}$ and $4^{\text {th }}$ ribs in dogs and cats. ${ }^{12}$ Its gross anatomical divisions comprise the right, left, caudate and quadrate lobes with their own blood supply and biliary drainage. The portahepatis transmits the hepatic artery, portal vein and right and left hepatic ducts (the portal triad), together with lymphatic and autonomic nerves. The venous drainage of the liver directly into the inferior vena cava comprises the right, left and middle hepatic veins, together with the small accessory hepatic veins. ${ }^{13}$ The anatomy of the liver differs between dogs and cats. Dogs have two separate ducts a pancreatic duct and a bile duct. In cats, however, the bile duct joins the pancreatic duct; this may allow the reflux and mixing of gastric secretion with both pancreatic secretions and bile. ${ }^{14}$

The internal structure of the liver is made small hexagonal functional units known as lobules. Each lobule consists of a central vein surrounded by hepatic portal veins and hepatic arteries. These blood vessels are connected by many capillary-like tubes called sinusoids which extends from the portal veins and arteries to meet the central vein like spokes on a wheel. Each sinusoid passes through liver tissue containing two main cell types including Kupffer cells and hepatoctes. ${ }^{10,15}$

Kupffer cells are a type of macrophages that passes through the sinusoids. Hepatocytes are liver cells which are cuboidal epithelial cells that line the sinusoids and make up the majority of cells in the liver. ${ }^{14,15}$ Canaliculi, tiny bile collection vessels run parallel to the sinusoids on the other side of the hepatocyte and drain into the bile ducts of the liver. ${ }^{15}$

\section{Physiology of the Liver}

The liver plays an active role in the process of digestion through the production of bile. ${ }^{16}$ When food containing fats reaches the duodenum, the cells of the duodenum release the hormone cholecystokinine to stimulate the gallbladder to release bile. Bile travels through the bile ducts and is released into the duodenum where it emulsifies large masses of fat. ${ }^{15}$

The worn out red blood cells called bilirubin, present in the bile is the product of liver's digestion. Kupffer cells in the liver catch and destroy old, worn out red blood cells and pass their components on to hepatocytes. ${ }^{16}$ They metabolized by hepatocytes into hemoglobin (the red oxygen-carrying pigment of red blood cells) into the components heme and globin. Globin protein is further broken down and used as an energy source for the body. The iron-containing heme group cannot be recycled by the body and is converted into the pigment bilirubin and added to bile to be excreted from the body. ${ }^{15-17}$ Bilirubin gives bile its distinctive greenish color. Intestinal bacteria further convert bilirubin into the brown pigment stercobilin, which gives feces their brown color. ${ }^{14,15}$

The hepatocytes have the important metabolic jobs that support the cells of the body because all of the blood leaving the digestive system passes through the hepatic portal vein. The liver is responsible for metabolizing carbohydrates, lipids, and proteins into biologically useful materials. ${ }^{15,17}$

Blood passes from the digestive organs through the hepatic portal circulation, the hepatocytes of the liver monitor the contents of the blood and remove many potentially toxic substances before they can reach the rest of the body. Enzymes in hepatocytes metabolize many of these toxins such as alcohols and drugs into their inactive metabolites. The liver also metabolizes and removes hormones from circulation produced by the body's own glands to maintain normal homeostasis of the body. ${ }^{15,19}$

The liver is storage organs of many essential nutrients, vitamins and minerals obtained from blood passing through the hepatic portal system. ${ }^{16}$ Glucose is transported into hepatocytes under the influence of the hormone insulin and stored as the polysaccharide glycogen, ${ }^{17}$ this maintain the blood glucose homeostasis. ${ }^{15,16}$ The liver cells also absorb and stores fatty acids from digested triglyceride's, ${ }^{15}$ vitamins such as vitamins A, D, E, K, and $\mathrm{B}^{12,17}$ the minerals iron and copper-in order to provide a constant supply of these essential substances to the tissues of the body. ${ }^{16,17}$

The liver is responsible for the production of several vital proteins components of prothrombin, fibrinogen and albumins. Prothrombin and fibrinogen proteins are coagulating factors involved in the formation of blood clots. Albumins are proteins that maintain the isotonic environment of the blood so that cells of the body do not gain or lose water in the presence of body fluids and it is carrier protein for carrying different chemicals, hormones, nutrients and cells for normal functioning of the body. ${ }^{16}$

The liver functions as an organ of the immune system through the function of the Kupffer cells that line the sinusoids. Kupffer cells are a type of fixed macrophage that form part of the mononuclear phagocyte system along with macrophages in the spleen and lymph nodes. The liver macrophages Kupffer cells play 
an important role by capturing and digesting bacteria, fungi, parasites, worn-out blood cells and cellular debris. ${ }^{16,19}$

\section{THE LIVER DISEASES IN DOGS AND CATS}

Any disorder that damages the liver is liver disease. Dogs and cats suffering from liver disease can be in serious danger, since the liver performs a number of important functions throughout the body. ${ }^{19}$ Liver disease is a problem occasionally occurred in older animals acquired diseases and in younger animals with both acquired and congenital disease. ${ }^{20}$ Hepatitis is familiarly termed as inflammation to the liver. It can be incurred by both infectious and non-infectious ways. ${ }^{21}$ It is very likely that more than one causative infectious agent may cause hepatitis in small animals. ${ }^{22}$

\section{The Liver Diseases Ethiopathophysiology}

Infectious hepatitis: The liver of small animals (dogs and cats), other animals and humans beings can be affected by a number of infectious agents like viruses, bacteria, parasites, fungus and protozoa. The liver cells affected by a number of infectious agents and results inability of the liver to function properly. ${ }^{22}$

Viral hepatitis: Inflammation of the liver by virus is named as viral hepatitis. It is very specific for each felines and canines. Infectious canine hepatitis is an acute liver infection in dogs caused by canine adenovirus type- $1 .{ }^{23}$ Infection with canine herpes virus causes an acute, rapidly fatal illness associated with hepatic necrosis vasculitis and/or immune-mediated mechanisms. ${ }^{24}$ Viruses that cause feline leukemia and feline infectious peritonitis can results in feline viral hepatitis, as the viruses destroy liver tissues. These pathogens not only destroy liver tissue but also affect other organs of the body. ${ }^{25}$

Parasites diseases: Infection with protozoan parasites Toxoplasma gondi and Leishmaniainfantum in cats and dogs cause chronic hepatitis by affecting kupper cells and hepatocytes in immune compromised patients. ${ }^{26}$ Infection with liver fluke Platynosomumconcinnum can cause acute and chronic cholangitis. Since the fluke infecting emerges from the intestine and migrate into the common bile duct, gallbladder, or hepatic ducts and cause hepatic bile duct damage. ${ }^{27}$

Bacterial diseases: Leptospirosis is caused by Leptospira Interrogans Serovars Icterohemorrbagic Ae and canicola which is the most common pathogenic bacteria that affect the liver of small animals. The disease produces an acute multisystem disease affecting the liver, kidney and other organs. It is known to cause hepatitis ${ }^{28}$ due to direct leptospiral cytotoxic effect on endothelial hepatocytic membranes. ${ }^{29}$ Infections in dogs and cats most commonly occur in immune compromised. It is an infectious disease characterized by necrosis of liver since the bacteria resides in cytoplasm of hepatocytes and results liver swelling with multiple areas of hepatocellular necrosis with infiltrates of neutrophils and mononuclear cells. ${ }^{30}$

Mycotic infection: The most common mycotic infections associated with liver dysfunction in small animals' are candidiasis is caused by Candidiaalbicans, histoplasmosis by Histoplasmacapsulatom; aspergilosis by Aspergilus fumigates. ${ }^{31}$ Fungal infections occurred in immune compromised patients. The spores from lungs or the intestines, or it may spread to other parts of the body through the bloodstream or lymphatic system, causing a generalized or systemic infection in different organs including liver. ${ }^{20}$

\section{Non Infectious}

Wilson's disease: Wilson's disease is anautosomal recessive inherited disease of copper metabolism. When the hepatic storage capacity of $\mathrm{Cu}$ is exceeded, parenchymal inflammation followed by cell death with $\mathrm{Cu}$ release into the plasma causing hemolysis. This causes an inability to excrete copper into the biliary tract, and results the formation and release of free copper which is toxic and since has the potential to create reactive oxygen species cause hepatocyte damage and subsequent chronic hepatitis and cirrhosis. ${ }^{32}$

Drugs and toxins The liver is the major site of drug metabolism and is therefore a common target of adverse drug reactions. Hepatotoxicity implies chemical-driven liver damage. Drug-induced hepatotoxicity is a significant cause of acute liver failure. Certain medicinal agents, when taken in overdoses and sometimes even when introduced within therapeutic ranges, may injure the organ. ${ }^{19}$ Other chemical agents, such as those used in laboratories and industries, natural chemicals and herbal remedies can also induce hepatotoxicity. Chemicals that cause liver injury are called hepatotoxins. ${ }^{6,19}$

Drugs can cause liver injury in several ways, three main types are usually referred dose-dependent (or intrinsic), dose-independent (or idiosyncratic metabolic) toxicity and drug allergy (or idiosyncratic immunological). These drugs have inherent ability to cause hepatic injury either by directs hepatocellular damage or by disturbance of hepatocellular homeostasis, which results in liver cell death. ${ }^{33}$

Drugs that cause liver damage in small animals (dogs and cats) are anticonvulsants drugs include primidone; phenytoin and phenobarbital have hepatotoxic effects, especially in long-term treatment. Antifungal ketoconazole and antibiotic (trimethoprimsulfa), antihelminths (mebendazole, diethylcarbamazine- oxybendazole, and thiacetarsamide), inhalation anesthetics (halothane and methoxyflurane) and analgesics (acetaminophen, naproxen, and phenylbutazone) cause toxicity on liver cells, Also certain environmental toxins (pesticides, herbicides, cleaning agents and plant toxins) have hepatotoxic effects in dogs and cats as well as other animals. ${ }^{22}$ The other hepatotoxicity include plant aflatoxins, cyanotoxins and mushroom toxins have direct hepatotoxic effect liver cells and cause acute liver failure. ${ }^{34}$

\section{Autoimmunity Hepatitis}

Autoimmune disorders results from an exaggerated reaction of the immune system directed against the body's own tissue. In artificial insemination by husband (AIH), the liver cells are no longer recognized as 'belonging' to organisms are there attacked by the immune system resulting in chronic inflammation of the liver. ${ }^{35}$ The immune reaction may be related to defects in the immunological control of auto-reactivity; with consequent loss of self-tolerance to liver auto-antigens. The aetiology of AIH remains unknown 
or idiopathic, but evidence suggests a coalescence of genetic susceptibility and environmental risks, female dogs and cats are more susceptible. $^{36}$

\section{Reactive Hepatitis}

Reactive hepatitis is an inflammatory disorder of the liver induced by an extra hepatic process. It is associated with disorders of many other organs apart from the liver including gastrointestinal, respiratory diseases, heart failure, diseases of the urinary and reproductive system. Different inflammatory mediators cytokines such as Interleukin-1 (IL-1), Interleukin-6 (IL-6) and Tumor Necrosis Factor- (TNF-) are released as lipopolysaccharide (LPS) can activate kupffer cells in the liver parenchyma. A consequence of this activation is the release of pro-inflammatory that induces leukocyte migration and therefore induces reactive hepatitis results excessive damage of liver cells. ${ }^{37}$

\section{Endocrine Disorder}

Endocrine diseases are imbalances in hormone levels. Hormone imbalances can affect pet's health in many ways. Endocrine diseases develop when the body produces too much hormone or too little hormone. Diabetes mellitus, hyperadrenocorticism (Cushing's disease), and hyperthyroidism can all cause impaired liver function because of their effects on the organ. ${ }^{38}$

Hyperthyroidism is one of the most common endocrine disorders which cause liver dysfunction. It is characterized by increased secretion of thyroid hormones T3 and/or T4. Excess T3 and/or T4 induce apoptosis of hepatoctes which is programmed cell death and cause liver dysfunction it is more common in cats but rare in dogs. ${ }^{39}$

Another endocrine disorder is Cushing's disease (hyperadrenocorticism). It is excessive production of cortisol hormone produced by the abnormalities adrenal glands. It is a common disease of adrenal hyper function that is seen most commonly in the dogs and rare in cats. ${ }^{40}$ In liver, cortisol induces enzymes activity and also decreases insulin from pancreas to then leading to hyperglycemia in Cushing's dogs and cats. Excessive liver metabolism causes the liver overload and the liver become hepatomegaly. ${ }^{18}$

\section{Bile Duct Obstruction}

Obstruction of the common bile duct is associated with a number of diverse primary conditions, including inflammation (e.g, pancreatitis, duodenitis, duodenal foreign body, etc.), cholelithiasis, gallbladder mucocele, cholecystitis, neoplsia, bile duct malformations, parasitic infection the bile duct and extrinsic compression, and fibrosis. ${ }^{19}$

Complete bile duct obstruction results cholestasis which is an impairment of bile flow from the liver to the duodenum. Bile cannot enter the distal "stagnant loop" of the ductal system or gallbladder (cystic duct occlusion). Increased ductal mucin contributes to duct distention. In this the biliary tree becomes colonized by bacteria cause cholangitis and ascending infection of the liver, inadequate antibiotic penetration into bile duct and results liver dysfunction from liver cell damage. ${ }^{41}$

\section{General Classification of Liver Diseases}

All diseases of hepatic parenchyma, vasculature or biliary tract are included as liver diseases. ${ }^{2}$ Hepatitis is inflammation of the liver, it can be acute and chronic. The most common liver diseases encountered in small animals include acute and chronic hepatitis. ${ }^{38}$

Acute hepatitis: Acute hepatitis is morphologically characterized by combination of inflammation hepatocellular apoptosis and necrosis and in some instances, regeneration. This can result in sudden death of the animal even within 48-hours after the start of the disease. $^{28}$

Chronic hepatitis: Chronic liver disease is also known as chronic hepatitis refers to a long-term pathological process of continuous destruction of liver parenchyma and its gradual substitution with fibrous tissue, which ultimately results in liver cirrhosis associated with a fatal outcome. ${ }^{42}$ It has structurally abnormal nodules (micro- or macro-nodules), it is considered irreversible, and usually it is idiopathic in origin. It is a regularly diagnosed condition in dogs, is less frequently encountered in cats but more frequently in dogs. ${ }^{43}$ Chronic liver dysfunction or injury is a serious health problem worldwide in animals and human beings. Chronic liver disease involves a wide range of liver pathologies that include hepatic lipidosis, neoplsia, fibrosis or cirrhosis, cholangitis complex. ${ }^{44}$

\section{Liver Diseases Pathophysiology}

Portosystemic shunt: A portosystemic shunt (PSS) is a congenital malformation within the blood supply to the liver. Blood coming from the digestive system is shunted from the portal circulation, around the liver effectively bypassing it. The implication of PSS is that toxins, such as ammonia, which would typically be removed by the liver can, accumulate in the systemic circulation leading to clinical signs including stunted growth and neurological symptoms. ${ }^{19,45}$

Hepatic lipidosis: Hepatic lipidosis (HL) is the accumulation of fat in the liver cells as fat is mobilized from the body stores for energy source. HL occurs in cats that become anorexic and lose significant amounts of weight. ${ }^{46}$

\section{Biliary Tract Abnormalities Dogs and Cats}

Cholangitis complex: Cholangitis (cholangiohepatitis) is inflammation of the biliary system and liver. It is the most common primary hepatic disorder in felines. The two main forms of cholangitis neutrophilic are cholangitis and lymphocytic cholangitis. Neutrophil cholangitis is thought to be caused by an ascending infection of the biliary tract and stemming from the intestine. Feline anatomy is thought to predispose to developing this condition. Pancreatitis and/or inflammatory bowel disease may occur along side neutrophilic cholangitis and may predispose to it. Lymphocytic cholangitis is thought to be immune-mediated; however, the aetiology is not known. ${ }^{11,47}$ 
Cholelithiasis: Cholelithiasis is medical condition resulting from the formation of stones in the gallbladder. ${ }^{48}$ Choleliths (gallbladder stones) are thought to form due to imbalances influxes between bile salts and cholesterol that maintain a liquid composition to bile. Resultant change to a thicker or congealed form of bile provides scaffolding for the deposition of cholesterol, bilirubin, or calcium salts resulting in the formation of choleliths. Other factors thought to promote cholelith formation include increased levels of gallbladder motility, bile stasis and biliary inflammation. ${ }^{49}$

Pneumobilia: The presence of gas in the biliary system is pneumobilia. It is a common finding in dogs and cats that have recently undergone biliary surgery or endoscopic biliary procedure, infection by gas forming bacteria (emphysematous cholangitis). ${ }^{50}$

Choledocholithiasis: It is condition when a gallstone lodged within any duct of the bile system. The ducts typically involved are the common bile duct, the cystic duct and the common hepatic duct. Gallstones usually form in the gallbladder. ${ }^{51}$

Cholecystitis: Cholecystitis means painful inflammation of the gallbladder most cases are caused by gallstones. Gallstone becomes stuck in the cystic duct (this is the tube that drains bile out from the gallbladder into the bile duct). Bile then builds up in the gallbladder which becomes stretched (distended). The walls of the gallbladder become inflamed. In some cases the inflamed gallbladder becomes infected. An infected gallbladder is more prone to lead to complications. $^{52}$

\section{Hepatic Neoplsia}

Neoplsia can occur in the liver either as a primary disease or due to metastases. The categories of hepatic tumors in cats and dogs are hepatocellular, bile duct and mesenchymal. ${ }^{18}$ In dogs, malignant tumors are more common, whereas benign neoplsia particularly cystic bile duct adenoma is more frequent in cats. Metastasis to the liver is most common from tumors of the spleen, pancrease, and gastrointestinal tract since the liver is rich in blood supply. Animals may have increased liver enzyme activities detected on serum biochemistry. ${ }^{53}$

\section{Mostly Encountered Clinical Symptoms of Liver Diseases Pathophysiology}

Hepatic encephalopathy: Hepatic encephalopathy can be acquired and congenital neurological changes that occur with liver failure as the liver becomes less able to remove toxins, drugs and metabolites from the blood. ${ }^{18}$ Ammonia is absorbed in the intestine and most of it is taken up by liver, where it is mostly converted to urea. An increased ammonia concentration in systemic circulation indicates the liver unable to adequately metabolize ammonia. It can also be caused by a defect in the urea cycle, or anomalies in portal circulation causing blood from the intestine to bypass the liver, as described on figure. ${ }^{54}$ It results abnormal mentation and neurologic dysfunction occurs as a result of exposure of the cerebral cortex to absorbed intestinal toxins that have not been removed from the portal circulation by the liver. ${ }^{18,19}$

Portal hypertension and ascites: Chronic liver disease increases re- sistance blood flow to the liver and results portal hypertension and the back flow of blood, lymphatic fluid and increased pressure in the portal system. From increased pressure in portal system there is leakage of blood from capillaries and causes abnormal fluid accumulation in abdomen cavity is as cites from low albumen production in the liver, organ failure like liver and heart. ${ }^{28,55}$

Jaundice (Ictrus): One of the most common symptoms of liver disease is jaundice which is yellowish tinge to the skin, in the eyes gums and ears. The liver is responsible for excreting bilirubin, by-product of red blood cell breakdown. When the liver isn't functioning this bilirubin builds up in the blood and leads to the yellowish appearance. The causes of jaundice are classified as prehepatic, hepatic, or post-hepatic in origin. Pre-hepatic jaundice occurs when red blood cell breakdown or hemolysis, produces bilirubin faster than the liver can metabolize it. Hepatic jaundice results from primary and seciseases within the liver that interfere with the liver cells' ability to metabolize bilirubin or excrete it normally into the biliary tract. Post-hepatic jaundice can result from obstruction to the flow of bilirubin-containing bile within the bile duct or from injury that causes leakage from the gallbladder or bile duct. ${ }^{20,46}$

Diagnosis of liver disease: Diagnosis of liver diseases can be difficult because the symptoms of the organ may be ambiguous or may easily interfere with the symptoms of other diseases. However, liver disease can be diagnosed by using history and clinical symptoms, liver function tests, medical imaging and tissue analysis or biopsy. ${ }^{2}$

Clinical symptoms: The liver has a large reserve capacity for many of the functions it carries out which results in relatively specific clinical indications of hepatobiliary diseases, such as icterus, ascites, coagulopathy or increases bleeding time, neurological signs and abdominal pain. ${ }^{41}$ In addition, early indications of a hepatobiliary disease, such as apathy, anorexia, polyuria, polydipsia, vomiting, weight loss, and anemia are highly non-specific and may occur as a result of diseases affecting many other organ systems. ${ }^{56}$

\section{Liver Function Tests Other than Liver Enzymes}

Liver function tests are helped to check livers health and detect liver damage. These tests known as liver chemistries to help to determine the health of liver by measuring the levels of proteins and bilirubin, bile acids, copper, cholesterol, glucose and complete blood count. ${ }^{57}$

Billirubin: An elevated level of bilirubin presence in the blood and urine indicates jaundice may become clinically evident as serum bilirubin level rises above the normal level in dogs $0.1-0.3 \mathrm{mg} / \mathrm{dL}$ and $0.1-0.4 \mathrm{mg} / \mathrm{dL}$ in cats. ${ }^{56,58}$

Bile acid: Bile acid concentrations elevation in dogs and cats are suggestive of hepatobiliary disease. Bile acid concentrations elevation from the normal range $25-30 \mu \mathrm{mol} / \mathrm{L}$ and $25 \mu \mathrm{mol} / \mathrm{L}$ in dogs and cats respectively are suggestive of hepatobiliary disease, i.e. decreased functional mass, alterations in portal circulation (or cholestasis). ${ }^{59}$

Albumin: Albumin is produced by hepatocytes it is released into 
the hepatic interstitial and subsequently into the sinusoids and hepatic veins. Hypoalbuminaemia is occurred on animals where the level of albumen in the serum is less than the normal in dogs 2.7-4.4 mg/L and 2.5-3.9 mg/L in cats. ${ }^{58}$ But hypoalbuminaemia usually is not specific to liver disease and can occur due to many other diseases the most important are protein-losing enteropathy, vasculitis, large exudative skin injuries and blood loss. ${ }^{60}$

Urea: Reduced blood urea nitrogen below normal range 6-25 mg/ $\mathrm{dL}$ in dogs and $14-36 \mathrm{mg} / \mathrm{dL}$ in cats indicates liver insufficiency. ${ }^{59}$ In the case of hepatic insufficiency (e.g., due to liver atrophy caused by a portosystemic shunt, PSS), urea levels may be reduced, as the liver cells are no longer capable of producing sufficient urea. ${ }^{61}$

\section{Cholesterol}

Cholesterol is one of several fatty substances (lipids) that circulate in pet's blood. Hypercholesterolemia may occur in conjunction with diseases associated with a cholestasis, there is a whole range of differential diagnoses for increased cholesterol values, while there are only a few causes of reduced cholesterol values (protein-losing enteropathy, certain malignant tumors and severe malnutrition). ${ }^{62}$ The elevation of cholesterol from the normal level 3-6.6 mmol/L in dogs and 1.8-4.2 $\mathrm{mmol} / \mathrm{L}$ in cats usually indicates liver disease. ${ }^{59}$

\section{Liver Function Test Based on Liver Enzymes}

Elevated liver enzymes may indicate inflammation or damage to liver cells. Inflamed or injured liver cells leak higher amounts liver enzymes into the bloodstream, which can result in elevated liver enzymes on blood tests. The elevated liver enzymes most commonly found are Alanine transaminase (ALT), Aspartate transaminase (AST), Alkaline phosphatase (ALP), Gamma-glutamyltranspeptidase (GGT) ${ }^{.63}$

Alanine aminotransferase: Alanine aminotransferase is an enzyme specific to the liver that is found in the cytoplasm of the liver cells. It is almost exclusively found within hepatocytes so serum ALT increase indicates high hepatocellular injury in dogs and cats (there is only a minor contribution from skeletal muscle and red blood cells). ALT can also increase mildly with muscular injury and gastrointestinal disease. The magnitude increase may relate to severity of liver damage but does not predict reversibility. ${ }^{64}$ In dogs and cats the normal range of ALT is $5-107 \mu / \mathrm{L}$ and $10-100 \mu / \mathrm{L}$ respectively. ${ }^{65}$

Alkaline phosphatase: It is primarily an indicator of cholestasis liver disease. It also increases with severe bone destruction and due to steroid induction. Hepatic ALP is found mainly in liver canalicular cell membranes and increases with biliary disease, especially with cholestasis. ${ }^{66}$ In dogs and cats the normal range of ALP is 10-150 $\mu / \mathrm{L}$ and $6-102 \mu / \mathrm{L}$ respectively. ${ }^{59}$

Gamma glutamyltransferase: Gamma glutamyltransferase (GGT) is found in many tissues, but serum GGT originates mainly in the liver. It is predominantly found in the intrahepatic biliary epithelial cells. Increases in GGT are most frequently observed in cholestatic liver diseases. In cats GGT often elevates in hepatic lipidosis where GGT elevation is uncommon in other liver diseases. In dogs GGT activity in liver disease is higher specificity and lower sensitivity for detection of hepatobiliary disease. ${ }^{66}$ In dogs and cats the normal range of GGT $0-14 \mu / \mathrm{L}$ and $1-10 \mu / \mathrm{L}$ respectively. ${ }^{61,64}$

Diagnostic imaging in liver diseases: Radiography and abdominal ultrasound are the most frequently used imaging modalities for assessment of the hepatobiliary system in dogs and cats, but alternative imaging techniques are now being used more frequently used are computed tomography and Endoscopic retrograde cholangiopancreatography. ${ }^{65}$

Ultrasonography: Abdominal ultrasonography (US) is considered the most practical diagnostic imaging procedure for detecting a hepatobiliary disease. US well-suited for liver imaging because it shows the alterations in the tissue structure as differences in echogenicity. This allows comparing liver tissue to other soft tissues, as well as detecting heterogeneous structures within the liver parenchyma. ${ }^{2}$ Hepatic lipidosis in cats could be diagnosed slightly more accurately than other diffuse hepatic diseases. ${ }^{60}$

Radiography: Radiography may be used in liver diagnostics mostly to evaluate the size and shape of the liver and possibly to locate the lesions more specifically. ${ }^{2}$ Abdominal radiographs allow assessment of hepatic size, shape, opacity and location in most patients. Radiography may also allow identification of extra hepatic abnormalities that affect the liver. ${ }^{66}$

Computed tomography (CT): It distinguishes mass lesions, detect changes in structure of hepatic parenchyma and the biliary system, identify choleliths, detect abnormal hepatic perfusion (involving the portal vein, hepatic artery or hepatic vein), and portal thrombi, and can give detail the extent of traumatic hepatobiliary injuries. ${ }^{2}$

Endoscopic retrograde cholangiopancreatography: Endoscopic retrograde cholangiopancreatography (ERCP) is advanced medical imaging a procedure that uses an endoscope and X-rays to look bile duct and pancreatic duct by inject a contrast medium, so they can be seen on radiographs. It is the latest technological method used primarily to diagnose and treat conditions of the bile ducts and main pancreatic duct, including gallstones as seen figure, inflammatory strictures (scars), leaks (from trauma and surgery) and cancer. $^{67}$

\section{Liver Biopsy}

The liver biopsy is the most accurate means to determine the necroinflammatory activity process. ${ }^{62}$ Liver biopsy and histology is the final examination in the diagnostic tree and provides a diagnosis of the lesions prognosis and for the selection of appropriate treatment, since liver biopsy is the only definitive way to obtain a specific diagnosis. ${ }^{67}$

It is obtained through the skin with a special needle and ultrasound guidance, or it may be obtained surgically under general anesthesia. ${ }^{66}$ Liver biopsy provides main histologic features in favors of liver disease lesion are: a histologic picture includes 
granulomas; substantial numbers of eosinophils, sharply stampedout perivenular necrosis, ground-glass-like hepatocytes, unusual vascular lesions, veno-occlusive lesions can be detected on eosine and hematoxylin staining procedures. ${ }^{62,67}$

Therefore, the diagnosis is usually based on pharmacological history; the relationship between drug intake and the onset of clinical signs. Another diagnosis is done by detecting the pathogen in clinical specimens and/or demonstrating an increase in antibody. ${ }^{68}$ Direct detection of the toxins in gastric content is confirmatory diagnosis. ${ }^{32}$

\section{TREATMENT AND MANAGEMENT OF LIVER DISEASES|}

Hepatic disease is often treatable and has a predictable prognosis when a definitive diagnosis is made. Anti-inflammatory drugs, antioxidants, diuretics, appropriate low protein diets, fluid therapies, antibiotics and protectants are mostly used for the treatment of liver disease and the latest advanced instrumental methods liver disease treatment. ${ }^{52,67}$

\section{Antioxidants and Choleretics}

Oxidation is a significant mechanism of hepatocellular damage; therefore providing antioxidants are free radical scavengers include vitamin $\mathrm{E}$, zinc, silymarin (milk thistle), $\mathrm{N}$-acetyl cistine (NAC) and SAMe. SAMe which increases hepatic and red blood cell glutathione levels is widely available as a nutraceutical in dogs and cats. It is helpful for treating toxic hepatopathies in humans and there is evidence for best efficacy the use of both SAMe and silymarin in dogs with acute toxic hepatopathies treatment. ${ }^{18,28}$

Choleretic stimulates bile flow indicated in all biliary stasis cases. The best choice choleretis is ursodeoxycholic acid (UDCA) and Metronidazole relies upon hepatic clearance. They are widely used in both human and veterinary. They displace toxic bile acids also important in immune modulating and encourage antioxidant activity. UDCA synergistic action with SAMe and vitamin E. It should be avoided in dogs with complete biliary obstruction, which is uncommon in dogs due to its potential to cause gallbladder rupture. ${ }^{14,28,67}$

\section{Diuretics and Gastro Protectants and Dietary Management}

It is important to check blood albumin levels, and if low, dietary control by supplementing the animal with a high biological value protein including cottage, cheese and fish are advised. ${ }^{28}$ The administration of blood products, e.g. canine plasma or human albumin solutions and also thiazide diuretics or furosemide can be initially used in combination with spironolactone to 'speed up' diuresis. ${ }^{14}$ Therapeutic paracentesis, which can cause a significant drop in blood albumin levels due to inability of the diseased liver to make up for the loss, should be avoided unless the ascites are life-threatening..$^{60,67}$

Portal hypertension is common in dogs with chronic hepatitis and leads to gut-wall oedema, which is at risk of ulceration. Perforation of GI ulcers causing septic peritonitis is a common cause of death in patients with chronic PH. Anorexia predisposes a patient to gastro intestinal ulceration; therefore, ensuring adequate enteral nutrition is important and supplementation ranitidine, Cimetidine and omeprazole are commonly ${ }^{63,66}$ Medications such as lactulose is required to reduce gut absorption of ammonia if the liver is too damaged to break. ${ }^{67}$ Zinc supplementation has been shown to reduce inflammation reduce copper absorption form the gut and to protect the liver. ${ }^{18,62}$

D-Penicillamine (DPA) is the most commonly used as chelator to treat hepatic copper accumulation and treatment is most effective in early stages of disease. A low-copper/highzinc diet can help to prevent accumulation or reaccumulation of hepatic copper in dogs with complex forms of copper-associated hepatitis 14. 2, 2, 2-tetramine tetrahydrochlodide may be more useful in acute hepatitis. ${ }^{68}$ Zinc gluconate or acetate can be used prophylactically, especially in young dogs known to have copper storage disease, to reduce the absorption of copper from the gastrointestinal tract (GI) and prevent the development of copperassociated hepatitis. ${ }^{28,67}$

\section{Antibiotics and Antifibrotics}

Ampillicin, cephalosporins, enrofloxacin, metronidazole and clindamycin and chloramphenicol are good choices antibiotics for treating bacterial liver diseases. ${ }^{10}$ Colchicines are a specific antifibrotics used in dogs with moderate to marked fibrosis. ${ }^{18,67}$

\section{Fluid Therapy}

Animals severely affected by liver disease, particularly those that are vomiting may require a period of hospitalization and intravenous fluids to help to flush out toxins from the blood stream and replace fluid lost in vomiting. ${ }^{68}$ Intravenous fluid include glucose, sodium salts and blood products as needed depending are required for treatment of liver disease in dogs and cats as well as human beings. ${ }^{34}$

\section{Latest Advanced Liver Disease Treatment}

Cholecystectomy: Cholecystectomy is surgical removal of the gallbladder. It is a common treatment of symptomatic gallstones and other gallbladder conditions. It is a laparoscopic cholecystectomy, and surgical on the gallbladder. ${ }^{67}$

Endoscopic retrograde cholangiopancreatography: Endoscopic retrograde cholangiopancreatography (ERCP), is an endoscopic procedure that can remove gallstones or prevent blockages by widening parts of the bile duct where gallstones frequently get stuck. ERCP is often used to retrieve stones stuck in the common bile duct with gallstone pancreatitis or cholangitis. ${ }^{65}$

Liver transplantation: A liver transplant is a surgical procedure that removes a liver that no longer functions properly (liver failure) and replaces it with a healthy liver. Liver transplant is usually reserved as a treatment option due to end-stage of chronic liver disease usually cirrhosis. ${ }^{63,68}$

\section{CONCLUSION AND RECOMMENDATIONS}

In pet liver disease is a broad term referring to any disorder that 
damages the liver. The liver performs a number of important functions throughout the body including the regulation of digestion and metabolism, the synthesis of hormones and proteins, immune response and filtering of toxins from the blood stream. The liver disease is mostly termed as hepatitis which is inflammation of the liver; it is acute and chronic. Infectious hepatitis can be occurred by virus, bacteria, fungus and parasites while noninfectious hepatitis is occurred by toxicity, endocrine disorders and bile duct obstruction, autoimmune hepatitis and reactive hepatitis. The development of the liver disease results neurological signs, ascites, vomiting, diarrhea, polyuria, polydipsia, weight loss, stunted growth, abdominal pain and changes the body fluids and as diseases progresses the liver is unable to function well and the dog and cat will die.

Based on the above conclusions the following recommendations for liver disease dogs and cats are forwarded: early accurate diagnosis of liver diseases of dogs and cats by using clinical symptoms and advanced diagnostic techniques. Based on diagnostic methods proper management and amelioration of the liver diseases of dogs and cats for maintaining good immunity and health must be required. For life threatened liver disease dogs and cats cholelithiasis surgical removal of gallbladder (cholecystectomy) or by Laparoscopy and common bile duct stone removal by ERCP. For cirrhotic liver dogs and cats liver transplantation is highly recommended.

\section{ACKNOWLEDGMENT}

I am highly indebted to AwiZone Nations and Nationalities Animal Health Department, Livestock Resource Development and Promotion Office; they give me.

\section{CONFLICTS OF INTEREST |}

The authors declare that they have no conflicts of interest.

\section{REFERENCES}

1. Prabhat S. Difference Between Cats and Dogs. 2018. Web site. http://www.differencebetween.net/science/nature/differencebetween-dog-and-cat/. Accessed on July 23, 2018.

2. Erina O. Difference between dogs and cats learn what makes each one special. 2018. Web site. http://erinollila.com//. Accesed on, June 9, 2012.

3. Somaye V, Paria P, Mozafar S. Anatomical and histological study of the liver and pancreas of two closely related mountain newts neurergusmicrospilotus and N.kaiseri (Amphibia: Caudata:n Salamandridae). J Vet Med. 2017; 34: doi: 10.3897/zoologia.34.e13229

4. Ramadori G, Moriconi F, Malik J, Dudas D. Physiology and pathophysiology of liver inflammation, damage and repair. J Physiol Pharmacol. 2008; 59 Suppl 1: 107-117.

5. Robert P, Favier A. Canine hepatitis and the pathomechanisms of Copper-induced Hepatitis in COMMD1 deficient dogs. Paper presented at: Dissertation Utrecht University; 2011; Utrecht, England.

6. Aashish P, Tarun S, Pallavi B. Drug-Induced Hepatotoxicity: A Review. Journal of Applied Pharmacentical Science. 2012; 2(5): 233-243. doi: 10.7324/JAPS.2012.2541

7. Ahmedullah F, Akbor M, Haider G, Ossain MK, Hossain A, Shanta L. Pathological investigation of liver of the slaughtered buffaloes in Barisal District. Bangle J Vet Med. 2008; 5(2): 81-85. doi: 10.3329/bjvm.v5i1.1321

8. Jason N. Indicators of liver disease. Journal of Small Animal Medicine. 2008; 8: 45.

9. Kumar V, Kumar A, Varshney AC, Tyagi SP, Kanwar MS, Sharma SK. Diagnostic, imaging of canine hepatobiliary affections: A review. J Vet Med Int. 2012; 67(107): 15. doi: 10.1155/2012/672107

10. Susan E. Liver disease and treatment in dogs and cats (Proceedings). 2018. Web site. http://veterinarycalendar.dvm360.com/ liver-disease-and-treatment-dogs-and-cats-proceedings. Accessed August 1, 2008.

11. Melissa S, Yonaire C. Hepatic encephalopathy: Diagnosis and treatment liver inflammation damage and repair. J Physiol Pharmacol. 2013; 59(1): 107-117.

12. Samantha F. The liver: Role in health and disease in small animals. 2013. Web site. https://www.vettimes.co.uk/app/uploads/ wp-post-to-pdf-enhanced-cache/1/the-liver-role-in-health-anddisease-in-small-animals.pdf. Accessed June 1, 2013.

13. Sant R.Approaches to diagnosis and treatment of hepatic disease. Vet Times. 2013; 43: 8-9.

14. Tim T. Anatomy and Physiology of Liver. 2018. Web site. http://www.innerbody.com/image_digeov/card10-new2.html. Accessed November 2, 2010.

15. Aspinall V, Cappello M. Introduction to Veterinary Anatomy and Histology. $2^{\text {nd }}$ ed. London, UK: Elsevier; 2009: 249.

16. Ozougwu JC. Physiology of the liver. International Journal of Research in Pharmacy and Biosciences. 2017; 4(8): 13-24.

17. Jennifer ES. Hepatobiliary diseases in dogs and cats. 2011.Web site. http://veterinarycalendar.dvm360.com/hepatobiliary-diseases-dogs-and-cats-proceedings?pageID=3. Accessed October 1, 2011.

18. Yuki U, Scott K, Andrew B. Diagnostic approach to gallbladder disease in dogs and cats. Journal of Companion Animal. 2017; 22(5): 254-260. doi: 10.12968/coan.2017.22.5.254

19. Joseph T. A Case-based Approach to the Canine Patient with Increased Liver. Enzymes (Parts 1 \& 2). 2017. Web site. http// www.scvsec.com. Accessed July 13, 2017. 
20. Pallavi K, Sravani D, Durga PN, Durga S, Pavan PN, Babu PS, Raviteja K. Hepatitis: A review on current and future scenario. J In Silico In Vitro Pharmacol. 2017; 3(1): 15. doi: 10.4172/24696692.100015

21. Bahman M, SalehE, Reza A. Dog with Clinical and HistopathologicalSigns of ICH (Infectious Canine Hepatitis). Iranian Journal of Veterinary Science and Technology. 2010; 2(2): 123-128.

22. Güra S, Acarb A. A retrospective investigation of canine adenovirus (CAV). Harold E. Anatomy of the Liver. $4^{\text {th }}$ ed. London, UK: Elsevier; 2011; 29.

23. Sharon A. Other Disorders of the Bile Ducts in Smal Animals. 2011. Web site. www.msdvetmanual.com/digestive-system/ hepatic diseasein-small-animals/other-disorders-of-the-bile-ductsin-small-animals. Accessed on May 10, 2018.

24. Osie FT. Hepatitis in Cats-Causes, Symptoms and Treatment. 2016. Web site. https://www.animalwised.com/hepatitis-in-catscauses-symptoms-and-treatment-411.htm. Accessed October 2, 2016.

25. Bouznach A, Edery N, Kelmer E, Shicaht N, Waner PS. Systemic toxoplasma gondii infection in a cat with incidental cholangioma. Israel Journal of Veterinary Medicine. 2015; 70(3): 2015.

26. Corma MA, Louis CP, Rothuizen JO. Feline biliary tree and gallbladder disease: Aetiology, diagnosis and treatment. Journal of Feline Medicine and Surgery. 2017; 9: 514-528. doi: 10.1177/1098612X17706465

27. Rothuizen J. General principles in the treatment of liver disease in small. Animals Journal Small Animal Medicine. 2010; 5(4): 78-89.

28. Pritt S, Henderson KS, Shek WR. Evaluation of available diagnostic methods for clostridium piliformein laboratory rabbits (Oryctolaguscuniculus). Journal Laboratory Animals. 2010; 44: 14-19. doi: 10.1258/la.2009.008079

29. Salgado CD, Martin EW. Fungal hepatitis in dogs and cats. Clinical Infectious Diseases. 2000; 31(2): 609-611.

30. Lionel S, Nicole S, Deon V, Merwe D, Schmid A. Liver failure in dog following suspected ingestion of blue-green algae (Microcystis spp.): A case report and review of the Toxin. J Am Anim Hosp Assoc. 2013; 49(39): 342-346. doi: 10.5326/JAAHA-MS-5913

31. Bantel H. Autoimmune Hepatitis. $2^{\text {nd }}$ ed. Germany: Falk foundation; 2017: 47.

32. Margaret C, Gideon M, Hirschfield H, David HA. Autoimmune hepatitis an approach to disease understanding and management. Br Med Bull. 2015; 114: 181-191. doi: 10.1093/bmb/ldv021

33. Stephan N, Wendy D. Reactive hepatitis in dogs. Global Veterinaria. 2012; 9(4): 454-459. doi: 10.5829/idosi.gv.2012.9.4.653
34. Saro K, Tse-Ling F. Hepatic dysfunction in hyperthyroidism. Journal of List Gastroenterology. 2011; 17(5): 20-23.

35. Chen G, Thomas KG. Interpretation of laboratory tests for canine Cushing's syndrome topical. Topics in Companion Animal Medicine. 2011; 26(2): 98-108. doi: 10.1053/j.tcam.2011.03.001

36. Tomohiro K, Nobuhiro O. Platelets in liver disease, cancer and regeneration. World J Gastroenterol. 2017; 23(18): 3228-3239.

37. Elhiblu MA, Dua K, Mohindroo J, Mahajan SK, Sood NK, Dhaliwal PS. Clinico-haemato-biochemical profile of dogs with liver cirrhosis. J Vet World. 2015; 8(4): 487-491. doi: 10.14202/vetworld.2015.487-491

38. Ming H, Li S, Tan HY, Wang N, Tsao S-W, Feng Y. Current status of herbal medicines in chronic liver disease therapy: The biological effects, molecular targets and future prospects. International Journal of Molecular Science. 2015; 29(35): 28705-45. doi: 10.3390/ ijms161226126

39. Nelson RW, Couto A. Diagnostic Tests for the Hepatobiliary System Small Animal Internal Medicine. $2^{\text {nd }}$ ed. Maryland: Mosby; 1998; 487509.

40. Jane AP. Feline Hepatic Lipidosis: Therapeutic Considerations. $2^{\text {nd }}$ ed. Mosby, Maryland. 2011; 459-500.

41. Pradhan MS, Dakshinkar NP, Wagaye UG, Bodkhe AM. Successful treatment of ascites of hepatic origin in $\operatorname{dog}$ and cats. Vet World. 2015; 1: 23.

42. Hideyuki K, Kenjiro F, Hajime T, AndKoichi O. Intrahepatic cholelithiasis dogs and cats. Canadian Vet J. 2017; 58(9): 971-973.

43. Wendy A. Veterinary cholelithiasis benign neglect or treatment. 2017. Web site. https://aspenmeadowvet.com. Accessed March 24, 2017.

44. Sherman SC, Tran H. Pneumobilia: Benign or life-threatening. J Emerg Med. 2006; 30(2): 147-153. doi: 10.1016/j.jemermed.2005.05.016

45. Lori. S. Choledocholithiasis. 2017. Web site. https://www.medicalnewstoday.com/articles/318941. Accessed August 14, 2017.

46. Nick I. Cholecystitis. 2016 Web site. www.bile/cholecystitis. Com. Accessed October 3, 2016.

47. Josep P, Marta P, Bachs L. Tumors diagnosis and treatment. Journal Veterinary Focus. 2010; 20(3): 47.

48. Hilla, J.M., Leisewitza, and B.R.andGoddarda, A. The utility of uric acid assay in dogs and cats as an indicator of functional hepatic mass. Journal South Afr Vet Ass. 2012; 82(2): 86-93.

49. Eleana G, Melanie D, Dimitrios P. Iron deficiency anemia in chronic liver disease: Etiopathogenesis, diagnosis and treatment. 
Ann Gastroenterol. 2017; 30: 405-413. doi: 10.20524/aog.2017.0152

50. Süleyman K, Ehsan S. Methods of diagnosing in liver diseases for dog and cats. Turkish Journal of Scientific Reviews. 2017; 10(2): 36-46.

51. Dechun F, Partha M, Juqiu A, Hua W. Inflammation in liver diseases. Mediators Inflamm. 2018; 2018: 3927134. doi: $10.1155 / 2018 / 3927134$

52. Pyleris EG, Dabos GK. Pathophysiology and management of acute liver inflammation. Vet J. 2010; 39(27): 134-145.

53. Kristiina R, Margo S. Liver Disease Testing. 2009. Web site. https://vcahospitals.com/know-your-pet/bile-acid-test. Accessed October 1, 2017.

54. Kerl ME, Cohn LA. Albumin in health and disease: Protein metabolism and function. A Companion Article on Causes and Treatment of Hypoalbuminemia. 2004; 940.

55. Ronald H. Cushing's Syndrome in Dogs. 2018. Web site. https://pets.webmd.com/dogs/cushings-syndrome-dogs\#1. Accessed July 7, 2018.

56. Kaneko JJ, Harvey JW, Bruse ML. Clinical Biochemistry of Domestic Animals. $6^{\text {th }}$ ed. San Diego, CA, USA: Academic Press; 2008 300-301.

57. Dirksen IA, Burgener J, Rothuizen TS, Vanden LC, Penning B, Spee A, et al. Sensitivity and specificity of plasma ALT, ALP, and bile acids for hepatitis in labrador retrievers. J Vet Int Med. 2017; 2(31): 1017-1027. doi: 10.1111/jvim.14716

58. Stockham SL, Scott MA.Fundamentals of Veterinary Clinical Pathology. $2^{\text {nd }}$ ed. Ames, Blackwel; 2008: 230.
59. Latimer KS, Mahaffey EA, Prasse KW. Duncan and Prasse's Veterinary Laboratory Medicine, Clinical Pathology. $4^{\text {th }}$ ed. Ames, IA, USA: Blackwell; 2003: 300-301.

60. Larson MM. The Liver and Spleen. Textbook of Veterinary Diagnostic Radiology. $5^{\text {th }}$ ed. St. Louis, USA: Saunders; 2007: 667-669.

61. Adler DG, Baron TH, Davila RE, Egan J, Hirota WK, Leighton JA, et al. Guidelines on the role of ERCP in diseases of the biliary tract and the pancreas. J Inet Vet Med. 2005; 13: 239.

62. Leonardo S. Liver biopsy in elevated liver functions tests? An old question revisited. J Hepatol. 2011; 35(2001): 290-294.

63. Bexfield N, Watson P. Treatment of canine liver disease 2. Managing clinical signs and specific liver diseases. In Practice Clinical Practice. 2009; 31: 130-135. doi: 10.1136/inpract.31.4.172

64. Daniel D. Continuing education in small animal common inflammatory liver diseases: Resident in small animal diagnostic imaging. Veterinary Ireland Journal. 2010; 6(11): 623-626.

65. Kim WR, Brown RS, El-Serage H. Burden of liver disease in the United States Summary of a workshop. J Am Vet. 2002; 8: 227-242.

66. Thapa BR, Anuj W. New diagnostic tests, liver function tests and their interpretation. Journal of Small Animal Medicine. 2007; 86(13): 560-569.

67. Wikipedia. Detailed diagram of an endoscopic retrograde cholangiopancreatograpy. 2017. Web site. https://en.wikipedia.org/ wiki/Cholecystectomy. Accessed October 10, 2018.

68. Clarke EA. Feline Hypertension: Risks, Diagnosis and Management. 2011. Web site. https://www.cathsplace.co.nz/naturalholistic-blogs-dogs-cats. Accessed June 4, 2018. 